\title{
Population Data on Finland $1900-1990^{1}$
}

\author{
UPDATED BY ANNELI MIETTINEN
}

\section{Resident population on 31 December}

\begin{tabular}{lccccr}
\hline & & \multicolumn{2}{c}{ Urban municipalities } & \multicolumn{2}{c}{ Rural municipalities } \\
& Whole country & number & $\%$ & number & $\%$ \\
\hline 1900 & 2655900 & 333400 & 12.5 & 2322600 & 87.5 \\
1910 & 2943400 & 432200 & 14.7 & 2511200 & 85.3 \\
1920 & 3147600 & 507400 & 16.1 & 2640200 & 83.9 \\
1930 & 3462700 & 715000 & 20.6 & 2747700 & 79.4 \\
1940 & 3695600 & 991700 & 26.8 & 2703900 & 73.2 \\
1950 & 4029800 & 1302400 & 32.3 & 2727400 & 67.7 \\
1960 & 4446200 & 1707000 & 38.4 & 2739200 & 61.6 \\
1970 & 4598300 & 2340300 & 50.9 & 2258000 & 49.1 \\
1980 & 4787800 & 2865100 & 59.8 & 1922700 & 40.2 \\
1981 & 4812200 & 2881300 & 59.9 & 1930900 & 40.1 \\
1982 & 4841700 & 2897100 & 59.8 & 1944600 & 40.2 \\
1983 & 4869900 & 2910500 & 59.8 & 1959400 & 40.2 \\
1984 & 4893700 & 2923800 & 59.7 & 1969900 & 40.3 \\
1985 & 4910700 & 2937900 & 59.8 & 1972800 & 40.2 \\
1986 & 4925600 & 3042900 & 61.8 & 1882700 & 38.2 \\
1987 & 4938600 & 3052400 & 61.8 & 1886200 & 38.2 \\
1988 & 4954400 & 3059500 & 61.8 & 1894800 & 38.2 \\
1989 & 4974400 & 3067000 & 61.7 & 1907400 & 38.3 \\
$1990^{*}$ & 4994600 & 3077900 & 61.6 & 1916700 & 38.4 \\
\hline & & & & &
\end{tabular}

Age structure

\begin{tabular}{rccccccccr}
\hline & \multicolumn{3}{c}{ Whole population } & \multicolumn{3}{c}{ Men } & \multicolumn{3}{c}{ Women } \\
& $0-14$ & $15-64$ & $65-$ & $0-14$ & $15-64$ & $65-$ & $0-14$ & $15-64$ & $65-$ \\
& $\%$ & $\%$ & $\%$ & $\%$ & $\% \%$ & $\%$ & $\%$ & $\%$ & $\%$ \\
\hline 1900 & 35.0 & 59.6 & 5.4 & 35.9 & 59.4 & 4.7 & 34.3 & 59.7 & 6.0 \\
1910 & 35.6 & 58.6 & 5.8 & 36.7 & 58.3 & 5.0 & 34.6 & 59.0 & 6.4 \\
1920 & 33.4 & 60.7 & 5.9 & 34.8 & 60.0 & 5.2 & 32.0 & 61.3 & 6.7 \\
1930 & 29.4 & 64.3 & 6.3 & 30.6 & 64.1 & 5.3 & 28.3 & 64.5 & 7.2 \\
1940 & 26.9 & 66.7 & 6.4 & 28.2 & 66.5 & 5.3 & 25.7 & 66.9 & 7.4 \\
1950 & 30.0 & 63.3 & 6.7 & 32.0 & 62.8 & 5.2 & 28.1 & 63.9 & 8.0 \\
1960 & 30.1 & 62.4 & 7.4 & 31.9 & 62.3 & 5.7 & 28.5 & 62.5 & 8.9 \\
1970 & 24.4 & 66.3 & 9.3 & 25.7 & 67.1 & 7.2 & 23.0 & 65.7 & 11.3 \\
1980 & 20.2 & 67.8 & 12.0 & 21.4 & 69.7 & 8.9 & 19.1 & 66.0 & 14.9 \\
1981 & 19.9 & 68.0 & 12.1 & 21.0 & 70.0 & 9.0 & 18.9 & 65.9 & 15.2 \\
1982 & 19.7 & 68.0 & 12.3 & 20.8 & 70.1 & 9.1 & 18.5 & 66.1 & 15.4 \\
1983 & 19.5 & 68.1 & 12.4 & 20.6 & 70.3 & 9.1 & 18.5 & 66.0 & 15.5 \\
1984 & 19.4 & 68.2 & 12.4 & 20.5 & 70.4 & 9.0 & 18.4 & 66.0 & 15.6 \\
1985 & 19.4 & 68.0 & 12.6 & 20.5 & 70.4 & 9.1 & 18.4 & 65.8 & 15.8 \\
1986 & 19.3 & 67.9 & 12.8 & 20.4 & 70.3 & 9.3 & 18.3 & 65.6 & 16.1 \\
1987 & 19.3 & 67.8 & 12.9 & 20.4 & 70.3 & 9.4 & 18.3 & 65.4 & 16.3 \\
1988 & 19.4 & 67.5 & 13.1 & 20.4 & 70.0 & 9.5 & 18.4 & 65.1 & 16.5 \\
1989 & 19.3 & 67.4 & 13.3 & 20.4 & 69.9 & 9.7 & 18.4 & 64.9 & 16.7 \\
\hline
\end{tabular}

1 The data are based on the statistics of the Central Statistical Office of Finland, Helsinki.

* Preliminary data 
Figure 1. Age structure in 1900, 1930, 1960, 1980 and 1989.

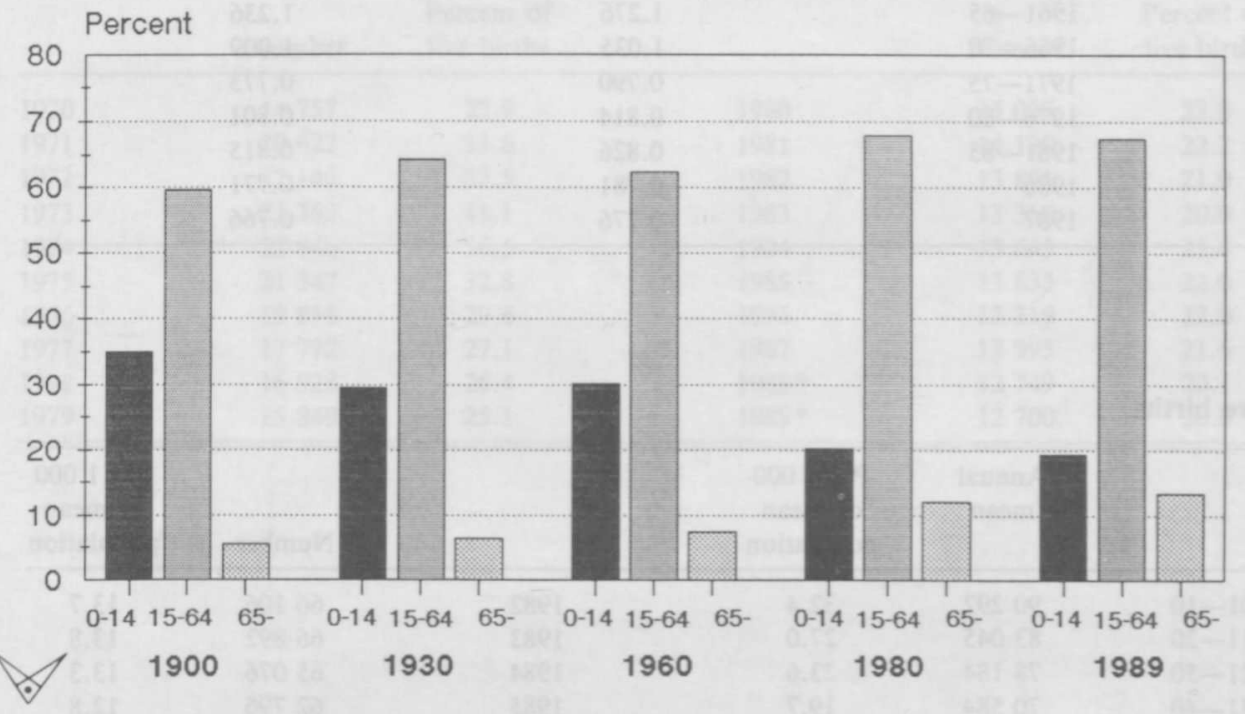

Age-specific fertility rate

\begin{tabular}{lrrrrrrrr}
\hline \multicolumn{7}{c}{} & \multicolumn{9}{c}{ Per 1 000 women } & & & $\begin{array}{c}\text { Total } \\
\text { fertility }\end{array}$ \\
& $15-19$ & $20-24$ & $25-29$ & $30-34$ & $35-39$ & $40-44$ & $45-49$ per woman \\
\hline $1901-10$ & 16.8 & 147.7 & 226.8 & 227.4 & 192.6 & 109.1 & 16.2 & 4.68 \\
$1911-20$ & 14.9 & 122.8 & 181.4 & 181.9 & 155.2 & 92.9 & 14.0 & 3.81 \\
$1921-30$ & 14.2 & 110.0 & 156.9 & 143.8 & 116.8 & 67.4 & 10.5 & 3.10 \\
$1931-40$ & 14.4 & 96.5 & 126.2 & 108.9 & 82.8 & 43.5 & 5.6 & 2.39 \\
$1941-45$ & 11.3 & 105.2 & 144.9 & 124.3 & 88.4 & 41.8 & 5.4 & 2.60 \\
$1946-50$ & 25.7 & 161.9 & 189.4 & 147.5 & 100.5 & 43.3 & 5.0 & 3.37 \\
$1951-55$ & 27.2 & 157.6 & 165.8 & 125.0 & 81.0 & 35.1 & 3.9 & 2.98 \\
$1956-60$ & 29.3 & 161.4 & 159.6 & 108.1 & 67.5 & 27.5 & 2.8 & 2.78 \\
$1961-65$ & 30.7 & 156.7 & 156.0 & 98.8 & 55.7 & 22.5 & 2.2 & 2.61 \\
$1966-70$ & 34.7 & 131.9 & 125.9 & 76.5 & 39.7 & 13.7 & 1.4 & 2.12 \\
$1971-75$ & 27.9 & 104.3 & 106.6 & 55.8 & 23.3 & 6.1 & 0.5 & 1.62 \\
$1976-80$ & 22.0 & 96.8 & 114.6 & 67.5 & 26.4 & 6.0 & 0.3 & 1.67 \\
$1981-85$ & 15.7 & 85.0 & 122.8 & 75.3 & 32.1 & 6.7 & 0.4 & 1.69 \\
1986 & 12.9 & 71.0 & 119.8 & 77.8 & 31.1 & 6.9 & .0 .4 & 1.60 \\
1987 & 12.1 & 67.8 & 119.1 & 80.1 & 31.0 & 7.8 & 0.3 & 1.59 \\
$1988 *$ & 12.3 & 70.4 & 128.3 & 86.4 & 33.4 & 7.7 & 0.5 & 1.70 \\
\hline
\end{tabular}

\section{Gross and net reproduction rate}

\begin{tabular}{ccc}
\hline & $\begin{array}{c}\text { Gross reproduction } \\
\text { rate }\end{array}$ & $\begin{array}{c}\text { Net reproduction } \\
\text { rate }\end{array}$ \\
\hline $1901-10$ & 2.278 & $\ldots$ \\
$1911-20$ & 1.849 & $\ldots$ \\
$1921-30$ & 1.504 & $\ldots$ \\
$1931-35$ & 1.167 & 0.958 \\
$1936-40$ & 1.162 & 1.048 \\
$1941-45$ & 1.262 & 1.469
\end{tabular}




$\begin{array}{lll}1951-55 & 1.452 & 1.373 \\ 1956-60 & 1.357 & 1.301 \\ 1961-65 & 1.276 & 1.236 \\ 1966-70 & 1.035 & 1.009 \\ 1971-75 & 0.790 & 0.773 \\ 1976-80 & 0.814 & 0.801 \\ 1981-85 & 0.826 & 0.815 \\ 1986 & 0.781 & 0.771 \\ 1987 & 0.776 & 0.766\end{array}$

\section{Live births}

\begin{tabular}{lccccc}
\hline & $\begin{array}{c}\text { Annual } \\
\text { mean }\end{array}$ & $\begin{array}{c}\text { Per } 1000 \\
\text { of mean } \\
\text { population }\end{array}$ & & & $\begin{array}{c}\text { Per } 1000 \\
\text { of mean } \\
\text { population }\end{array}$ \\
\hline $1901-10$ & 90292 & 32.4 & 1982 & 66106 & 13.7 \\
$1911-20$ & 83045 & 27.0 & 1983 & 66892 & 13.8 \\
$1921-30$ & 78184 & 23.6 & 1984 & 65076 & 13.3 \\
$1931-40$ & 70584 & 19.7 & 1985 & 62796 & 12.8 \\
$1941-50$ & 92613 & 24.3 & 1986 & 60632 & 12.3 \\
$1951-60$ & 88024 & 20.7 & 1987 & 59827 & 12.1 \\
$1961-70$ & 76466 & 16.8 & $1988^{*}$ & 63314 & 12.8 \\
$1971-80$ & 62789 & 13.3 & $1989^{*}$ & 63355 & 12.8 \\
1981 & 63469 & 13.2 & $1990^{*}$ & 65639 & 13.2 \\
\hline
\end{tabular}

Median age of mother at first birth

\begin{tabular}{lccc}
\hline & Median age & & Median age \\
\hline $1961-65$ & 23.25 & 1983 & 25.34 \\
$1966-70$ & 22.73 & 1984 & 25.46 \\
$1971-75$ & 24.03 & 1985 & 25.66 \\
$1976-80$ & 25.18 & 1986 & 25.86 \\
1981 & 25.41 & 1987 & 26.14 \\
1982 & 25.17 & $1988^{*}$ & 26.24 \\
\hline
\end{tabular}

\section{Illegitimate live births}

\begin{tabular}{lccccc}
\hline & $\begin{array}{c}\text { Annual } \\
\text { mean }\end{array}$ & $\begin{array}{c}\text { Percent } \\
\text { of all live } \\
\text { births }\end{array}$ & Number & $\begin{array}{c}\text { Percent } \\
\text { of all live } \\
\text { births }\end{array}$ \\
\hline $1901-10$ & 6120 & 6.8 & 1981 & 8431 & 13.3 \\
$1911-20$ & 6573 & 6.8 & 1982 & 9007 & 13.6 \\
$1921-30$ & 6606 & 8.4 & 1983 & 9386 & 14.0 \\
$1931-40$ & 5349 & 7.6 & 1984 & 9825 & 15.1 \\
$1941-50$ & 5629 & 6.1 & 1985 & 10292 & 16.4 \\
$1951-60$ & 3819 & 4.3 & 1986 & 11467 & 19.0 \\
$1961-70$ & 3621 & 4.7 & & & 19.2 \\
$1971-80$ & 6182 & 9.8 & & & \\
\hline
\end{tabular}


Abortions

\begin{tabular}{lccccc}
\hline & Number & $\begin{array}{c}\text { Percent of } \\
\text { live births }\end{array}$ & & Number & $\begin{array}{c}\text { Percent of } \\
\text { live births }\end{array}$ \\
\hline 1970 & 14757 & 22.9 & 1980 & 15037 & 23.8 \\
1971 & 20622 & 33.8 & 1981 & 14120 & 22.2 \\
1972 & 22146 & 37.5 & 1982 & 13861 & 21.0 \\
1973 & 23362 & 41.1 & 1983 & 13360 & 20.0 \\
1974 & 22846 & 36.5 & 1984 & 13645 & 21.0 \\
1975 & 21547 & 32.8 & 1985 & 13833 & 22.0 \\
1976 & 19818 & 29.6 & 1986 & 13319 & 22.0 \\
1977 & 17772 & 27.1 & 1987 & 12995 & 21.6 \\
1978 & 16928 & 26.4 & $1988^{*}$ & 12749 & 20.1 \\
1979 & 15849 & 25.1 & $1989^{*}$ & 12700 & 20.0 \\
\hline
\end{tabular}

\section{Marriages}

\begin{tabular}{lccccc}
\hline & $\begin{array}{c}\text { Annual } \\
\text { mean }\end{array}$ & $\begin{array}{c}\text { Per } 1000 \\
\text { of mean } \\
\text { population }\end{array}$ & & Number & $\begin{array}{c}\text { Per } 1000 \\
\text { of mean } \\
\text { population }\end{array}$ \\
\hline $1901-10$ & 18947 & 6.8 & 1982 & 30459 & 6.3 \\
$1911-20$ & 18922 & 6.2 & 1983 & 29474 & 6.1 \\
$1921-30$ & 23725 & 7.2 & 1984 & 28550 & 5.8 \\
$1931-40$ & 28491 & 7.9 & 1985 & 25751 & 5.3 \\
$1941-50$ & 37367 & 9.5 & 1986 & 25820 & 5.2 \\
$1951-60$ & 32191 & 7.6 & 1987 & 26259 & 5.3 \\
$1961-70$ & 37398 & 8.2 & $1988^{*}$ & 26453 & 5.3 \\
$1971-80$ & 32575 & 6.9 & $1989^{*}$ & 25043 & 5.0 \\
1981 & 30100 & 6.3 & $1990^{*}$ & 24150 & 4.8 \\
\hline
\end{tabular}

Median age at first marriage

\begin{tabular}{lcclll}
\hline & Men & Women & & Men & Women \\
\hline $1901-05$ & 26.1 & 23.5 & $1961-65$ & 24.1 & 22.3 \\
$1906-10$ & 26.2 & 23.6 & $1966-70$ & 23.6 & 22.2 \\
$1911-15$ & 26.4 & 23.7 & $1971-75$ & 24.0 & 22.7 \\
$1916-20$ & 27.0 & 24.0 & $1976-80$ & 25.2 & 23.3 \\
$1921-25$ & 26.9 & 24.0 & 1981 & 25.8 & 23.8 \\
$1926-30$ & 26.6 & 23.9 & 1982 & 26.0 & 24.0 \\
$1931-35$ & 27.1 & 24.4 & 1983 & 26.1 & 24.1 \\
$1936-40$ & 27.6 & 24.7 & 1984 & 26.4 & 24.4 \\
$1941-45$ & 27.6 & 24.3 & 1985 & 26.6 & 24.5 \\
$1946-50$ & 26.1 & 23.7 & 1986 & 26.8 & 24.9 \\
$1951-55$ & 24.9 & 23.2 & 1987 & 26.8 & 25.0 \\
$1956-60$ & 24.6 & 22.8 & 1988 & 27.0 & 25.2 \\
\hline
\end{tabular}


Figure 2. Median age at first marriage.

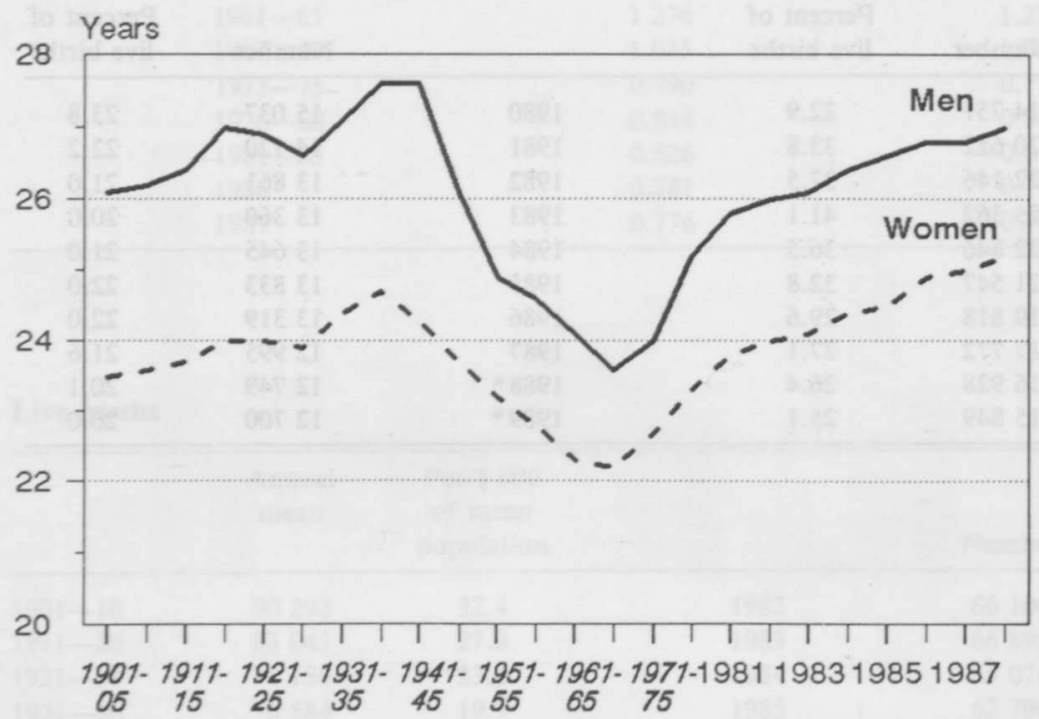

\section{Divorces}

\begin{tabular}{lccccc}
\hline & $\begin{array}{c}\text { Annual } \\
\text { mean }\end{array}$ & $\begin{array}{c}\text { Per 1 000 } \\
\text { of mean } \\
\text { population }\end{array}$ & Number & $\begin{array}{c}\text { Per 1 000 } \\
\text { of mean } \\
\text { population }\end{array}$ \\
\hline $1951-60$ & 3584 & 0.84 & 1984 & 9652 & 1.97 \\
$1961-70$ & 4860 & 1.06 & 1985 & 9064 & 1.85 \\
$1971-75$ & 8727 & 1.87 & 1986 & 9742 & 1.98 \\
$1976-80$ & 10051 & 2.12 & 1987 & 10110 & 2.05 \\
1981 & 9497 & 1.98 & $1988^{*}$ & 13307 & 2.69 \\
1982 & 9705 & 2.01 & $1989^{*}$ & 14558 & 2.93 \\
1983 & 9770 & 2.01 & $1990^{*}$ & 13169 & 2.64 \\
\hline
\end{tabular}

\section{Consensual unions}

\begin{tabular}{cccccccc}
\hline & $\begin{array}{c}\text { Proportion }(\%) \\
15-24\end{array}$ & of married couples and consensual unions & & Persons living in \\
& $25-29$ & $30-34$ & $35-44$ & $45-64$ & All & consensual union \\
\hline 1981 & 44.6 & 19.4 & 12.4 & 6.5 & 2.5 & 10.3 & 214100 \\
1985 & 55.0 & 27.1 & 13.5 & 7.4 & 4.4 & 12.3 & 283000 \\
1987 & 62.5 & 32.9 & 15.2 & 9.6 & 4.7 & 14.8 & 324000 \\
\hline
\end{tabular}

Families with children under 18 years of age by type of family and number of children (percent)

Married couples with children*

$\begin{array}{cc} & 1 \\ 1960 & 34.4 \\ 1970 & 40.2 \\ 1975 & 44.9 \\ 1978 & 44.9 \\ 1980 & 44.6 \\ 1982 & 44.6 \\ 1984 & 43.2\end{array}$

$2 \quad 3$

$$
30.7
$$

33.9

37.0

39.8

41.1

41.3

41.7

\section{3}

17.5

15.5

12.6

11.5

11.1

11.1

12.0
$4+$

17.4

10.4

5.5

3.8

3.2

3.0

3.2
Number

601542

602076

593346

595944

588373

581316

569568 


$\begin{array}{rrr} & 3+ \\ 42.4 & 41.9 & 15.6 \\ 41.1 & 42.3 & 13.0\end{array}$

Mothers with children

\begin{tabular}{|c|c|c|c|c|c|}
\hline 23 & 1 & 2 & 3 & $4+$ & Number \\
\hline 1960 & 61.1 & 23.0 & 9.2 & 6.7 & 67381 \\
\hline 1970 & 60.7 & 23.8 & 9.5 & 6.0 & 66303 \\
\hline 1975 & 62.4 & 25.7 & 8.3 & 3.6 & 72359 \\
\hline 1978 & 66.2 & 25.2 & 6.5 & 2.1 & 90292 \\
\hline 1980 & 67.7 & 25.3 & 5.6 & 1.4 & 88896 \\
\hline 1982 & 69.8 & 24.4 & 4.8 & 1.1 & 90150 \\
\hline 1984 & 70.1 & 24.4 & $\begin{array}{l}4.6 \\
3+\end{array}$ & 0.9 & 88419 \\
\hline 1985 & 70.0 & 24.5 & 5.5 & & 87409 \\
\hline 1987 & 69.0 & 25.2 & 4.8 & 1.0 & 86377 \\
\hline \multicolumn{6}{|c|}{ Fathers with children } \\
\hline & 1 & 2 & 3 & $4+$ & Number \\
\hline 1960 & 56.7 & 24.8 & 10.3 & 8.2 & 9123 \\
\hline 1970 & 61.1 & 24.3 & 9.0 & 5.6 & 8656 \\
\hline 1975 & 64.0 & 24.6 & 8.4 & 3.0 & 10386 \\
\hline 1978 & 68.7 & 23.9 & 5.6 & 1.8 & 10909 \\
\hline 1980 & 71.1 & 23.0 & 4.8 & 1.1 & 11463 \\
\hline 1982 & 73.9 & 21.9 & 3.6 & 0.6 & 11399 \\
\hline 1984 & 74.9 & 21.3 & $\begin{array}{l}3.3 \\
3+\end{array}$ & 0.6 & 11573 \\
\hline 1985 & 74.1 & 22.0 & 4.2 & & 11677 \\
\hline 1987 & 73.8 & 22.0 & 3.7 & 0.6 & 11918 \\
\hline
\end{tabular}

* incl. not-married couples with children under 18 years

Figure 3. Families with children under 18 years of age.

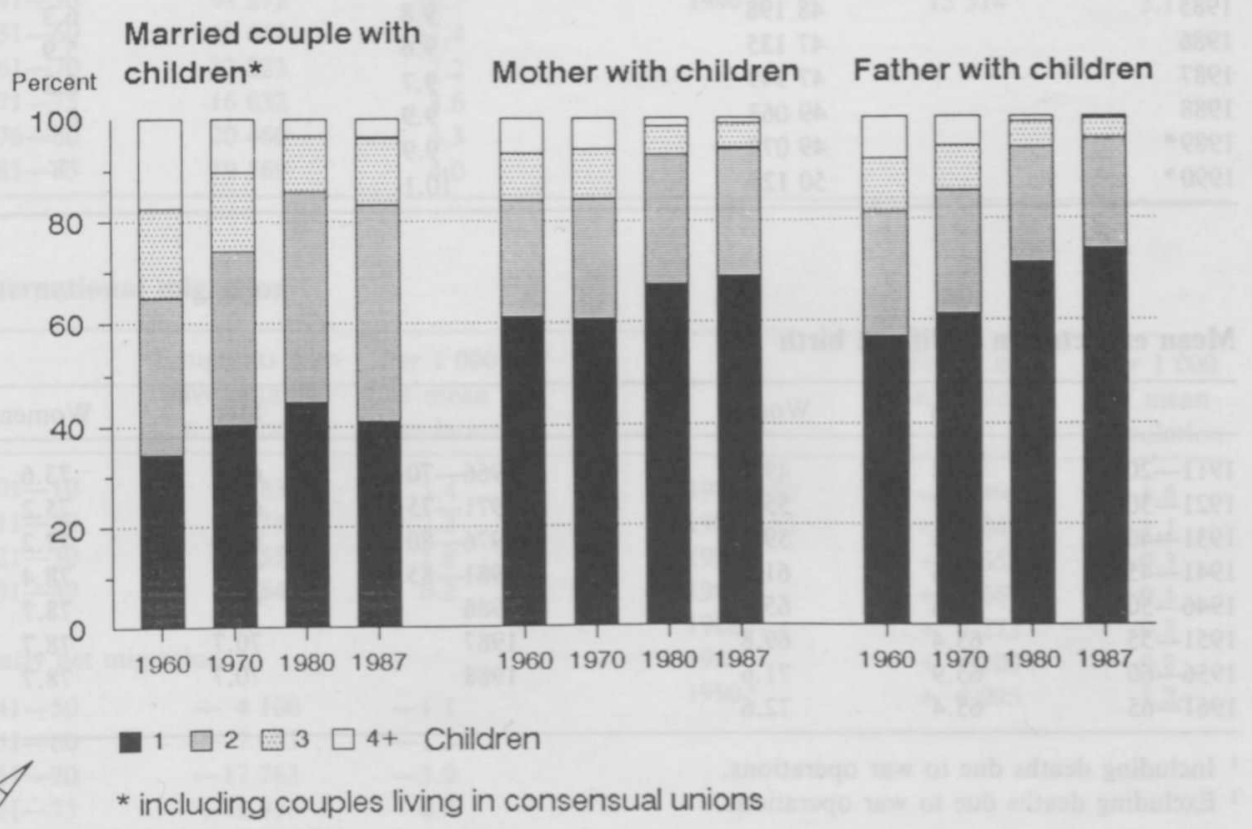


Families with children under 18 years of age by type of family

\begin{tabular}{ccccc}
\hline & $\begin{array}{c}\text { Married couples } \\
\text { with children* } \\
\%\end{array}$ & $\begin{array}{c}\text { Mothers } \\
\text { with children } \\
\%\end{array}$ & $\begin{array}{c}\text { Fathers } \\
\text { with children } \\
\%\end{array}$ & Number \\
\hline 1960 & 88.7 & 10.0 & 1.3 & 678046 \\
1970 & 88.9 & 9.8 & 1.3 & 677035 \\
1975 & 87.8 & 10.7 & 1.5 & 676091 \\
1978 & 85.4 & 13.0 & 1.6 & 697145 \\
1980 & 85.4 & 12.9 & 1.7 & 688732 \\
1982 & 85.1 & 13.2 & 1.7 & 682865 \\
1984 & 85.1 & 13.2 & 1.7 & 659870 \\
1985 & 85.0 & 13.2 & 1.8 & 643993 \\
1987 & 84.7 & 13.4 & 1.9 & \\
\hline
\end{tabular}

* incl. not-married couples with children under 18 years

\section{Deaths}

\begin{tabular}{lccc}
\hline & $\begin{array}{c}\text { Annual mean/ } \\
\text { Number }\end{array}$ & $\begin{array}{c}\text { Per 1 000 } \\
\text { of mean } \\
\text { population }\end{array}$ & $\begin{array}{c}\text { Infant mortality } \\
\text { per } 1 \text { 000 live } \\
\text { births }\end{array}$ \\
\hline $1901-10$ & 52099 & 18.7 & 123.8 \\
$1911-20$ & 58284 & 19.0 & 111.8 \\
$1921-30$ & 49573 & 14.9 & 91.9 \\
$1931-40$ & 50222 & 14.0 & 72.0 \\
$1941-50$ & 51838 & 13.6 & 56.0 \\
$1951-60$ & 39381 & 9.3 & 28.6 \\
$1961-70$ & 43494 & 9.5 & 16.7 \\
$1971-80$ & 44243 & 9.4 & 11.3 \\
1981 & 44404 & 9.3 & 6.5 \\
1982 & 43408 & 9.0 & 6.0 \\
1983 & 45388 & 9.3 & 6.2 \\
1984 & 45098 & 9.2 & 6.5 \\
1985 & 48198 & 9.8 & 5.9 \\
1986 & 47135 & 9.6 & 6.2 \\
1987 & 47949 & 9.7 & 6.1 \\
1988 & 49063 & 9.9 &.. \\
$1989^{*}$ & 49072 & 9.9 & \\
$1990^{*}$ & 50125 & 10.1 & \\
\hline
\end{tabular}

Mean expectation of life at birth

\begin{tabular}{lccccc}
\hline & Men & Women & & Men & Women \\
\hline $1911-20^{1}$ & 43.4 & 49.1 & $1966-70$ & 65.9 & 73.6 \\
$1921-30$ & 50.7 & 55.1 & $1971-75$ & 66.7 & 75.2 \\
$1931-40^{2}$ & 54.5 & 59.6 & $1976-80$ & 68.5 & 77.2 \\
$1941-45^{2}$ & 54.6 & 61.1 & $1981-85$ & 70.1 & 78.4 \\
$1946-50$ & 58.6 & 65.8 & 1986 & 70.5 & 78.7 \\
$1951-55$ & 63.4 & 69.8 & 1987 & 70.7 & 78.7 \\
$1956-60$ & 65.9 & 71.6 & 1988 & 70.7 & 78.7 \\
$1961-65$ & 65.4 & 72.6 & & & \\
\hline
\end{tabular}

1 Including deaths due to war operations.

2 Excluding deaths due to war operations. 
Figure 4. Mean expectation of life at birth.

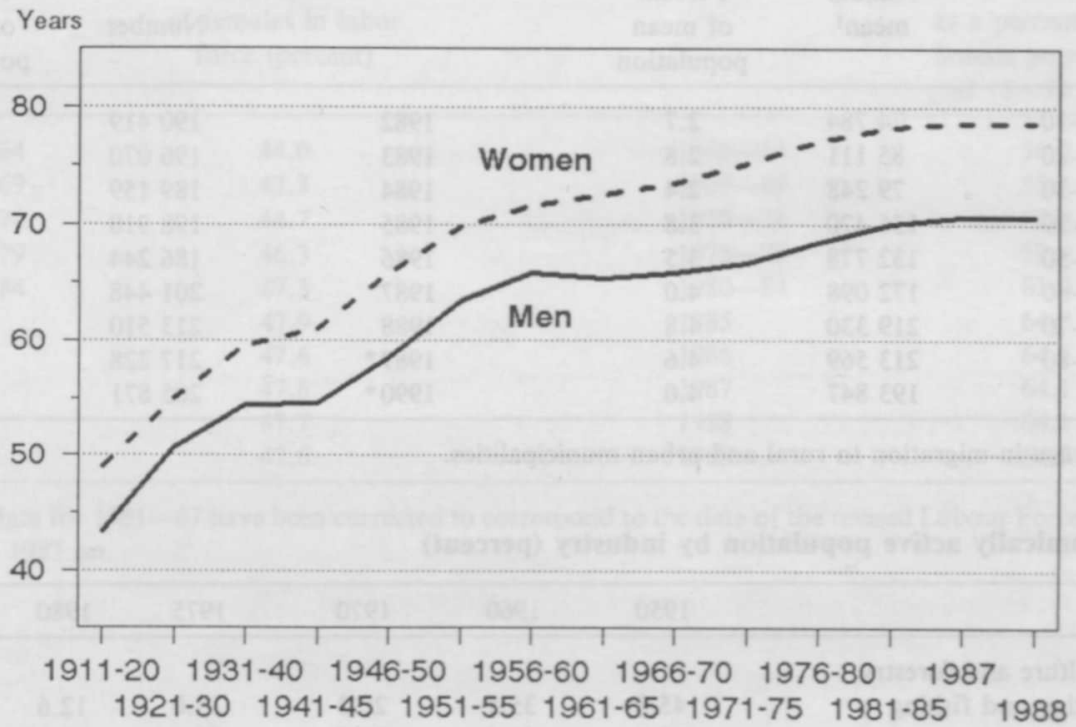

Excess of births over deaths

\begin{tabular}{lccccc}
\hline & Annual mean & $\begin{array}{c}\text { Per } 1000 \\
\text { of mean } \\
\text { population }\end{array}$ & & Number & $\begin{array}{c}\text { Per } 1000 \\
\text { of mean } \\
\text { population }\end{array}$ \\
\hline $1901-10$ & 38194 & 13.7 & 1986 & 13497 & 2.7 \\
$1911-20$ & 24760 & 8.1 & 1987 & 11878 & 2.4 \\
$1921-30$ & 28611 & 8.7 & $1988^{*}$ & 14251 & 2.9 \\
$1931-40$ & 20354 & 5.7 & $1989^{*}$ & 15514 & 3.9 \\
$1941-50$ & 44272 & 10.7 & & & \\
$1951-60$ & 48643 & 11.4 & & & \\
$1961-70$ & 32223 & 7.2 & & & \\
$1971-75$ & 16632 & 3.6 & & & \\
$1976-80$ & 20460 & 4.3 & & & \\
$1981-85$ & 19569 & 4.0 & & & \\
\hline
\end{tabular}

\section{International migration}

\begin{tabular}{|c|c|c|c|c|c|}
\hline & $\begin{array}{c}\text { Emigrants who } \\
\text { have obtained } \\
\text { a passport }\end{array}$ & $\begin{array}{l}\text { Per } 1000 \\
\text { of mean } \\
\text { population }\end{array}$ & & $\begin{array}{l}\text { Yearly net } \\
\text { migration }\end{array}$ & $\begin{array}{c}\text { Per } 1000 \\
\text { of mean } \\
\text { population }\end{array}$ \\
\hline $1901-10$ & 150832 & 5.4 & \multirow{9}{*}{$\begin{array}{l}1976-80 \\
1981-85 \\
1986 \\
1987 \\
1988^{*} \\
1989^{*} \\
1990^{*}\end{array}$} & \multirow{9}{*}{$\begin{array}{r}-7493 \\
+5347 \\
+1658 \\
+\quad 667 \\
+1273 \\
+4200 \\
+6095\end{array}$} & \multirow{9}{*}{$\begin{array}{r}-1.6 \\
1.1 \\
0.3 \\
0.1 \\
0.3 \\
0.8\end{array}$} \\
\hline $1911-20$ & 67346 & 2.2 & & & \\
\hline $1921-30$ & 58559 & 1.8 & & & \\
\hline $1931-40$ & 8844 & & & & \\
\hline \multicolumn{2}{|c|}{ Yearly net migration } & & & & \\
\hline $1941-50$ & -4100 & -1.1 & & & \\
\hline $1951-60$ & -7003 & -1.5 & & & \\
\hline $1961-70$ & -17763 & -3.9 & & & \\
\hline $1971-75$ & +1936 & 0.4 & & & \\
\hline
\end{tabular}


Internal migration

\begin{tabular}{lccccc}
\hline & $\begin{array}{c}\text { Annual } \\
\text { mean }\end{array}$ & $\begin{array}{c}\text { Percent } \\
\text { of mean } \\
\text { population }\end{array}$ & & Number & $\begin{array}{c}\text { Percent } \\
\text { of mean } \\
\text { population }\end{array}$ \\
\hline $1901-10$ & 74784 & 2.7 & 1982 & 190419 & 3.9 \\
$1911-20$ & 85111 & 2.8 & 1983 & 196070 & 4.0 \\
$1921-30$ & 79248 & 2.4 & 1984 & 189159 & 3.9 \\
$1931-39$ & 136470 & 3.8 & 1985 & 198910 & 4.1 \\
$1941-50$ & 132778 & 3.5 & 1986 & 186244 & 3.8 \\
$1951-60$ & 172098 & 4.0 & 1987 & 201448 & 4.1 \\
$1961-70$ & 219330 & 4.8 & 1988 & 213510 & 4.3 \\
$1971-80$ & 213569 & 4.6 & $1989^{*}$ & 217228 & 4.4 \\
1981 & 193847 & 4.0 & $1990^{*}$ & 205871 & 4.1 \\
\hline
\end{tabular}

${ }^{1}$ Average in-migration to rural and urban municipalities.

Economically active population by industry (percent)

\begin{tabular}{lrrrrrr}
\hline & 1950 & 1960 & 1970 & 1975 & 1980 & $1985^{1}$ \\
\hline Agriculture and forestry, & & & & & & \\
hunting and fishing & 45.8 & 35.5 & 20.3 & 15.0 & 12.6 & 10.6 \\
Industry etc. & 20.8 & 21.6 & 25.9 & 27.2 & 26.3 & 24.3 \\
Construction & 6.3 & 8.7 & 8.3 & 8.5 & 7.1 & 7.4 \\
Commerce & 9.5 & 13.6 & 18.9 & 19.3 & 19.1 & 21.3 \\
Transport and communications & 5.4 & 6.3 & 7.1 & 7.6 & 7.9 & 7.6 \\
Services & 10.8 & 14.0 & 18.1 & 20.9 & 24.8 & 28.5 \\
Unknown & 1.4 & 0.3 & 1.4 & 1.5 & 2.2 & 0.3 \\
\hline & 100.0 & 100.0 & 100.0 & 100.0 & 100.0 & 100.0 \\
\hline
\end{tabular}

${ }^{1}$ Employed labor force

Figure 5. Economically active population by industry.

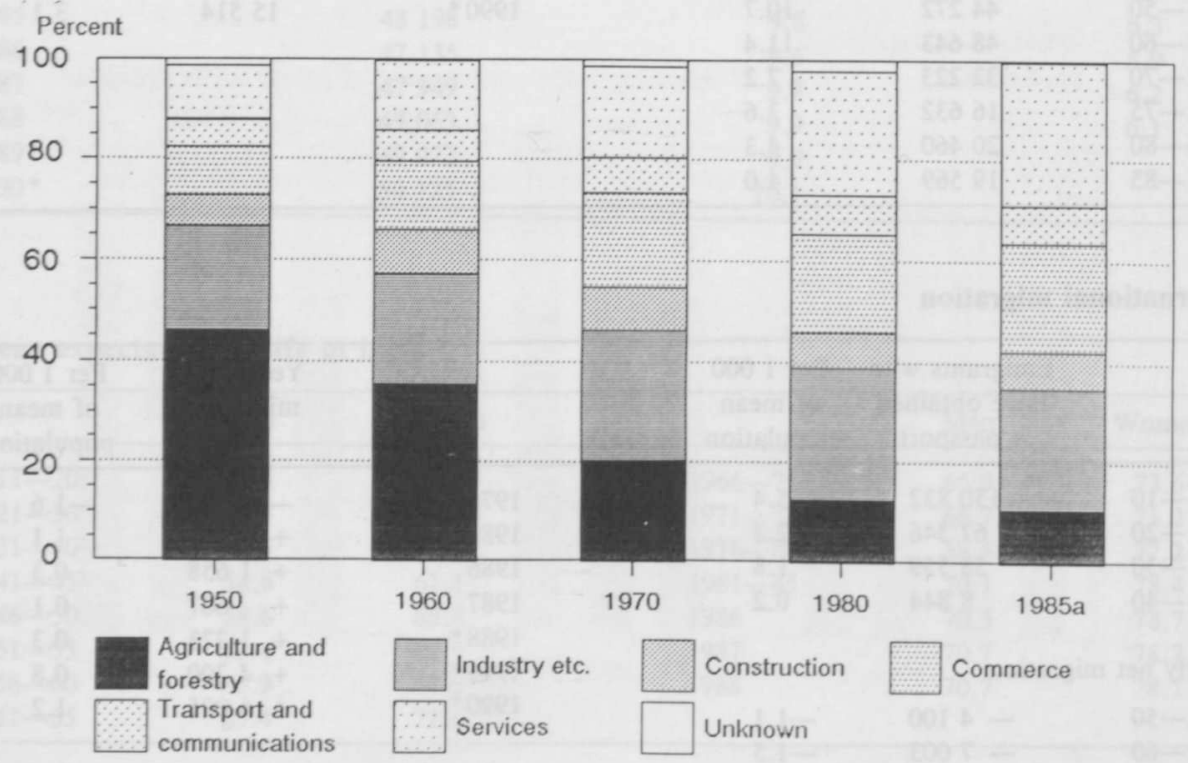


Females in labor force ${ }^{1}$

\begin{tabular}{lccc}
\hline & $\begin{array}{c}\text { The proportion } \\
\text { of females in labor } \\
\text { force (percent) }\end{array}$ & & $\begin{array}{r}\text { Female labor force } \\
\text { as a percentage of } \\
\text { female population } \\
\text { of 15-74 years }\end{array}$ \\
\hline $1960-64$ & 44.0 & $1960-64$ & 56.2 \\
$1965-69$ & 43.3 & $1965-69$ & 55.1 \\
$1970-74$ & 44.7 & $1970-74$ & 57.9 \\
$1975-79$ & 46.3 & $1975-79$ & 59.9 \\
$1980-84$ & 47.3 & $1980-84$ & 63.0 \\
1985 & 47.9 & 1985 & 64.8 \\
1986 & 47.6 & 1986 & 64.5 \\
1987 & 47.6 & 1987 & 64.1 \\
1988 & 47.7 & 1988 & 64.1 \\
\hline
\end{tabular}

${ }^{1}$ The data for 1981 - 87 have been corrected to correspond to the data of the revised Labour Force Survey from 1987 on. 